\title{
الجوانب العصبية للغة في تعلم اللغة العربية
}

\section{Hellen Tiara, Nur Khomisah Pohan, Yuli Imawan}

Uin Sunan Kalijaga Yogyakarta

\section{hellentiara1001@gmail.com.nurkhomisahpohan@gmail.com.}

yuliimawan5@gmail.com

\section{الملنخص}

اللغة نقطة انفصال أساسية للبشر عن بقية الكائنات الحية؛ حتى إن أكثر الكائنات قدرة على التواصل لا يبلغ في إمكاناته قدرات طفل في الثانية من عمره، يتعلم الأطفال اللغة بمجرد متابعة ذويهم ومن دون الحاجة لتدريب مسبق فقط لأن لهم القدرة على ذلك، حتى إن الأطفال الصم إذا لم يعلمهم أحد لغة الإشارة فسوف يبتكرون لغة إشارة خاصة بكم للتواصل مع الآخرين. يدرس علم اللغة العصبي علاقة اللغة والتواصل بالعناصر المختلفة لوظائف الدماغ، وبعبارة أخرى فهو يحاول أن يستكشف كيفية فهم الدماغ للغة والتواصل وانتاجهما. تابة هذه المقالة باستخدام طريقة البحث في المكتبة. البيانات المستخدمة كمصدر للكتابة هي في شكل كتب ومقالات والعديد من مصادر البيانات أو المعلومات الأخرى التي تعتبر ذات صلة بالدراسة. وفي الوقت نفسه، في تحليل البيانات، يستخدم الكاتب طريقة التحليل الوصفي.

\section{الكلمات المفتاحية: الجوانب العصبية للغة، تعلم اللغة العربية}

Keywords: Neurological aspects of language, learning Arabic

أ. أ. خلفية

يتطلب الطلب على تعلم اللغة العربية بشكل أساسي تحسين حالة متعلمي الدماغ في تلقي

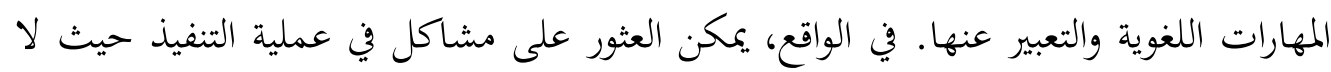
يمكن أن تتحقق مهارة التحدث كمهارة لغوية أساسية بشكل جيد، عند فحصها بشكل خاص من خلال منظور علم النفس العصبي. وذلك لأن تصميمات الغرض والمواد لا تأخذ في الاعتبار احتياجات الدماغ للمتعلمين. بالإضافة إلى ذلك، يميل اختيار طرق التدريس والاستراتيجيات إلى عكس تحسين الدماغ الأيسر على الرغم من أن اللغة تكمن في الدماغ الأيسر. على العكس من ذلك، إذا لم يتم تكبير الدماغ وتوازنه بشكل جيد، فإن تعلم اللغة الذي يتطلب الإبداع المتجسد في الدماغ الأيمن لا يمكن تنشيطه بشكل جيد أيضًا. 
لتقدم في التكنولوجيا والعلوم، مما أحدث تأثيرًا وتأثيرًا هائلين لتطوير الدراسات العلمية

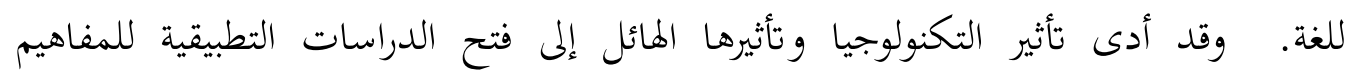

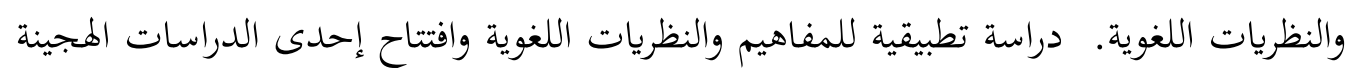

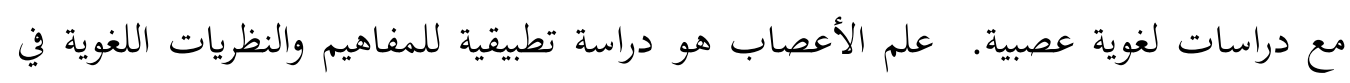

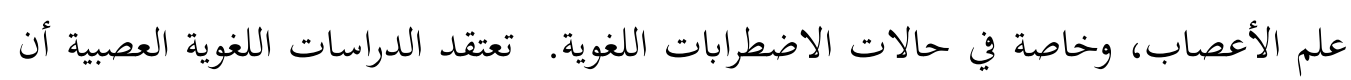

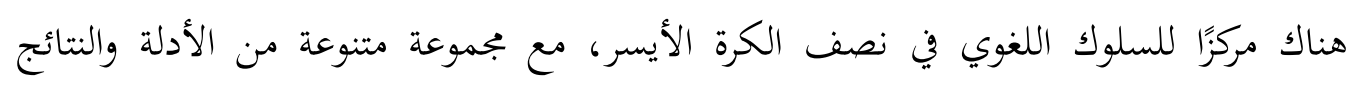

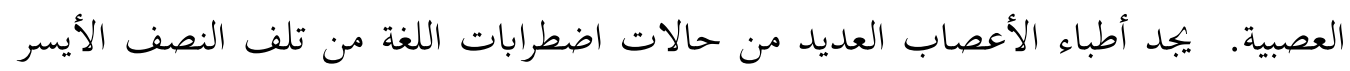

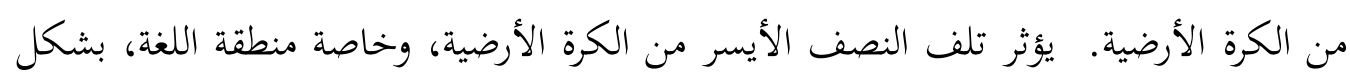
كبير على سلوك اللغة. لا يؤثر نفس الضرر على نصف الكرة الأيمن، على السلوك اللغوي. من المتوقع أن يساعد تطبيق المفاهيم والنظريات اللغوية. 1

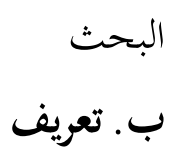

علم اللغة العصبي: تعريفه وبجال دراسته تضم عبارة علم اللغه العصبي "Neourolinguistik"

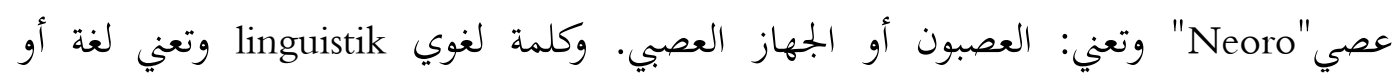

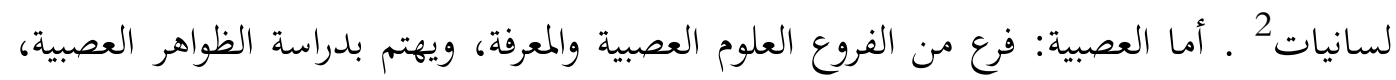
التي ترتبط او تتحكم في اللغة وهي: فهم اللغة، انتاج اللغة اكتساب اللغة.

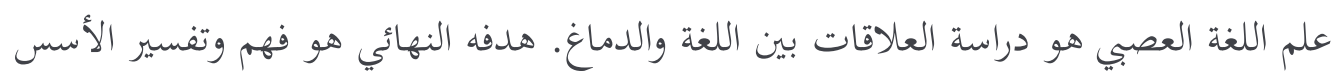
العصبية لمعرفة اللغة واستخدامها.

يدرس علم اللغة العصبي علاقة اللغة والتواصل بالعناصر المختلفة لوظائف الدماغ، وبعبارة

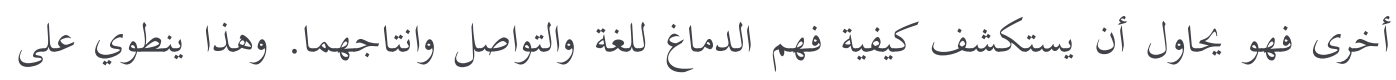

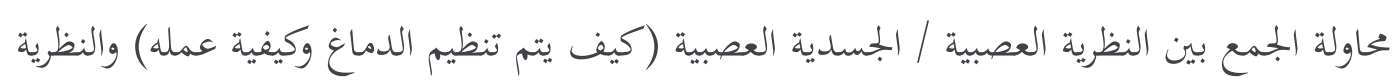

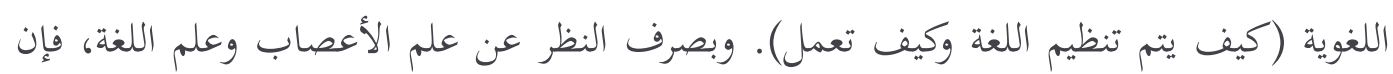

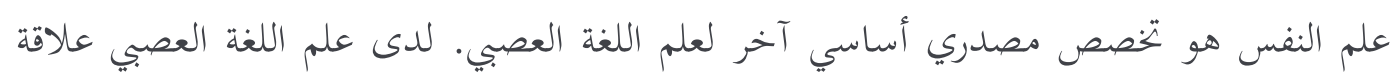

${ }^{1}$ New Horizons in The Study of Language and Mind. America: Cambridge University Press. Glezerman, Tatyana, Victoria Balkoski. 2002.

$$
\text { 22 - تدريب الرارى ص 5-6، نزهد النظر ص }
$$


وثيقة بعلم اللغة النفسي، لكنه يركز أكثر على دراسات الدماغ3. ربما تكون الدراسات حول اللغة

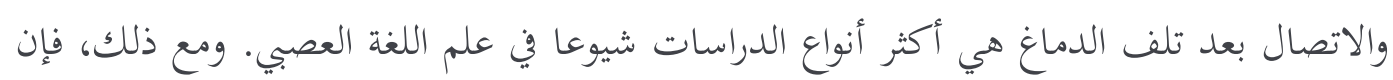

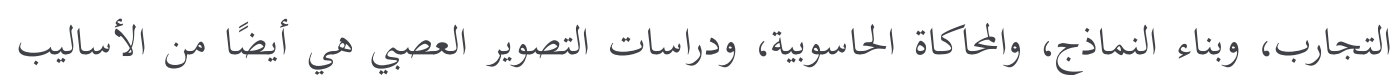

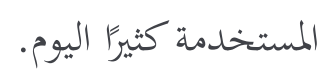

من أجل تحديد النماذج والأطر الملائمة لعلم اللغة العصبي، دعونا أولاً نحاول تحديد مجال البحث هذا. لقد تمت معالجة الأسئلة الرئيسية التي قم علم اللغة العصبي (انظر القسم الذي يحمل هذا العنوان أدناه) لأول مرة منذ فترة طويلة. كانت هناك فترة من التركيز المكثف في أواخر القرن

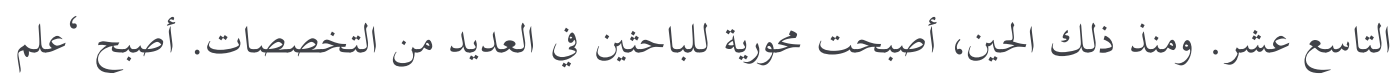
اللغة العصبي' مصطلحًا ثابتًا للحقل في الستينيات، تحت تأثير ثورة تشومسكي في علم اللغة وتطور علم اللغة النفسي كحقل محدد. لقد تم وصف موضوع علم اللغة العصبي في مقدمة سلسلة المجلدات المعروفة باسم 'دراسات في علم اللغة العصبي(Studies in Neurolinguistics) '، والتي قام بتحريرها ويتاكر وويتيكر Whitaker and Whitaker) في السبعينيات، على النحو التالي: على الرغم من أن مجال علم اللغة العصبي متعدد التخصصات بشكل صريح، إلا أن هناك موضوعًا مشتركًا للعلاقات بين اللغة والدماغ (Whitaker and Whitaker).

\section{ج. الجوانب العصبية للغة}

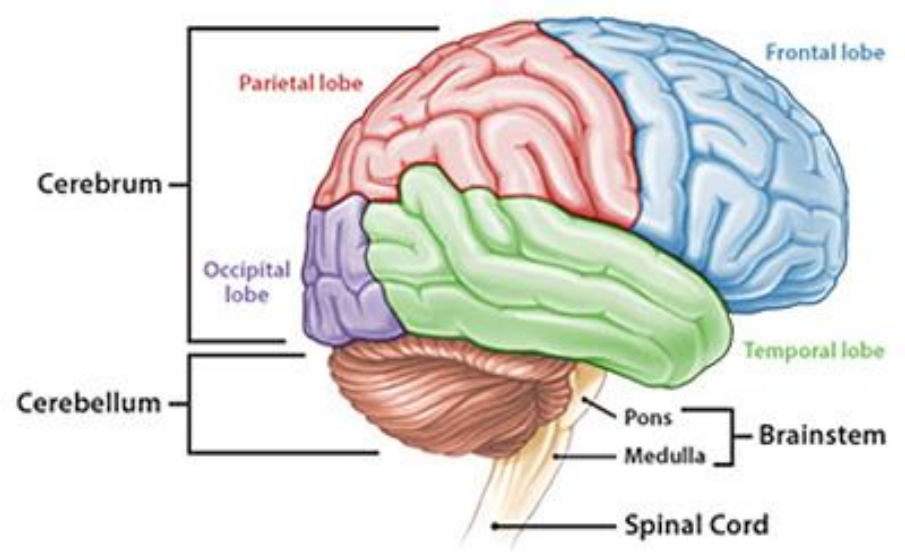

غرام لكنها تحتوي على حوالي 100 مليار خلية عصبية (خلايا عصبية). بشكل

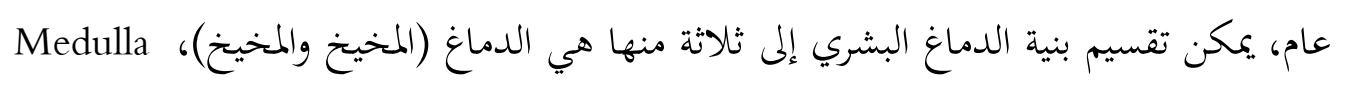
4 Spinalis Cord و Spinalis

$$
3 \text { حمد بن علويالمالكى الحسني. القوعد الأساسية في علم مصطلح الحلديث }
$$

4 الثعاليب، أبو منصور، فقه اللغة وسر العربية، حتقيق: عبد الرزاق املهدي، إحياء الرتاث العرب، ط1 12 | الطموحات - السنة الثالثة، العدد الثاني، أغسطس 2020 available at: http://journal.uir.ac.id/index.php/THUMUHAT 
1. أجزاء من الدماغ، وهي المخيخ (المخيخ)، المخيخ (المخيخ). جذع الدماغ (Brainstem) الأيسر، الفص الجبهي، الفص الجداري، الفص القذالي، الفص الصدغي. الجزء من المخ الذي يشارك مباشرة في معالجة اللغة هو القشرة الدماغية. القشرة الدماغية هي الجزء الذي يبدو وكأنه كتل بيضاء اللون وهو الجزء الأكبر في نظام الدماغ البشري. ينظم هذا القسم أو يدير العمليات المعرفية لدى البشر، وأحدها بالطبع اللغة.

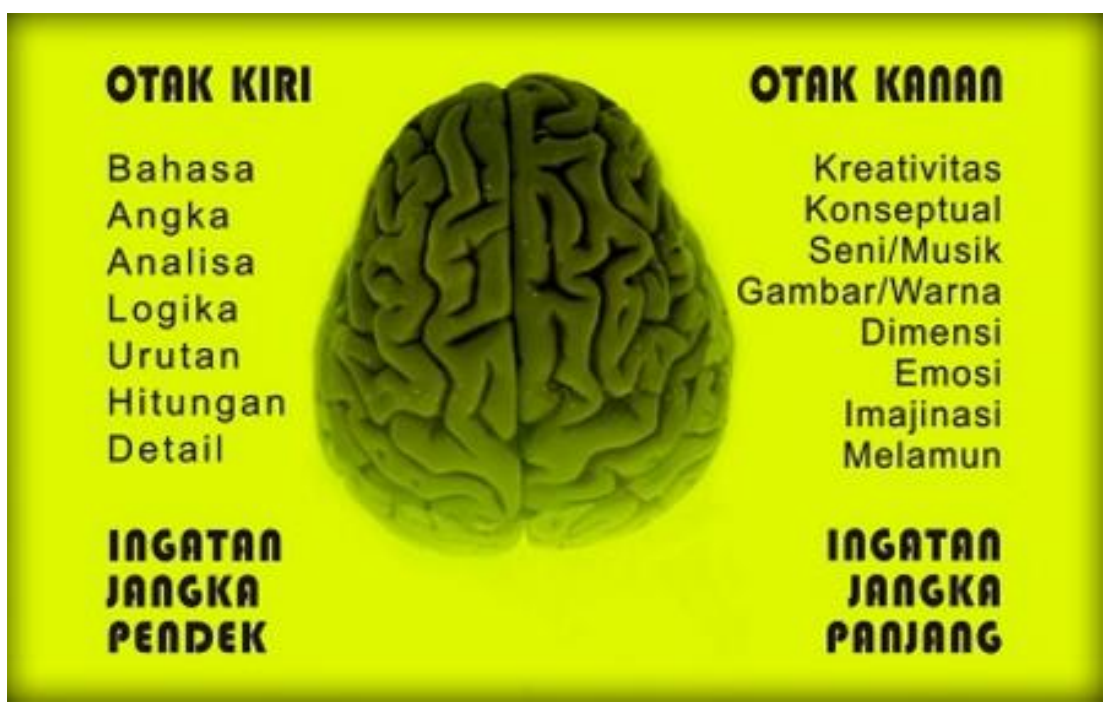

يتكون الدماغ من جزأين، هما نصف الكرة الأيسر (نصف الكرة الأيسر) ونصف الكرة الأيمن (نصف الكرة الأيمن). يتحكم نصف الكرة الأيمن في معالجة المعلومات المكانية والبصرية (رؤية أو تقدير أو فهم الفضاء أو الأشياء في ثلاثة أبعاد). بينما يتحكم نصف الكرة الأيسر في الأنشطة المعرفية واللغوية الأخرى. التنسيق بين الاثنين ممكن بسبب البنية التي توحد نصفي الكرة الأرضية، وهما الجسم الثفني 2. وظيفة الدماغ أي الوظيفة الأولية هي الحركة، والشعور، والحواس الخمسة، والوظائف المعقدة عقلية. نبيلة، قشرية، أفكار، ذكريات، عواطف. الإدراك والتنظيم وتنظيم الحركة، وظيفة الكام (اللغة). 3. تطور الدماغ وفقا لـ (Volpe, 1987) هو تشكيل أنابيب عصبية جديدة rofilerasi) لتشكيل الخلايا العصبية والخلايا الدبقية. إزاحة الخلايا الخلوية من جرثومة subemememal 
إلى القشرة، التمايز الحلوي لخلايا عصبية محددة، وتطوير محاور عصبية وتغضنات تتسبب في

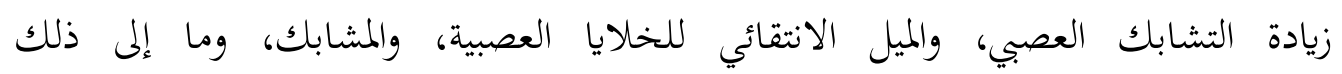
للمواصفات

4. تتكون الوظيفة اللغوية للدماغ من نصف الكرة الأيسر ونصف الكرة الأيمن، ونصفها

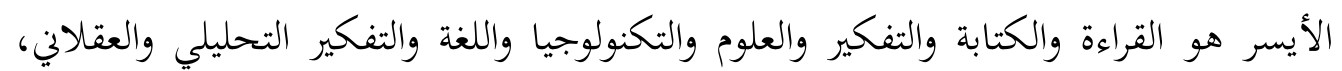

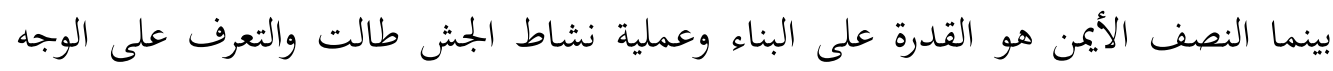
وخطوط الصورة. معقدة، والموسيقى والأغاني، والتعابير اللغوية التلقائية.

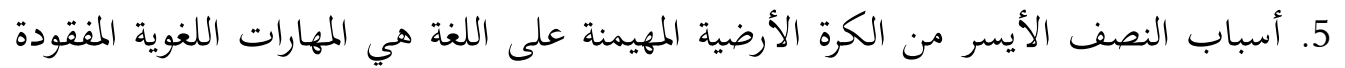

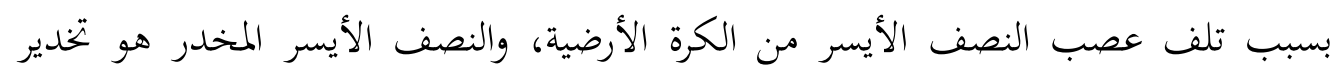

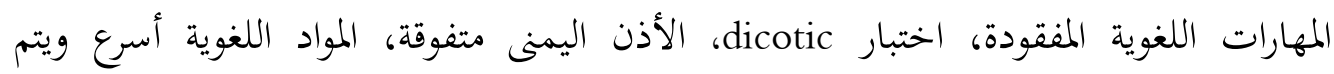

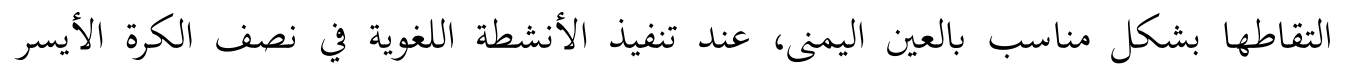

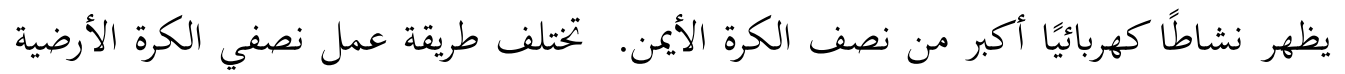
في كل شخص. 6. نظرية التحويل الجانبي هي اختبار استماع مزدوج، اختبار تتفيز كهربائي، اختبار رسومي اللنشاط الكهربائي، اختبار وادا، تقنية فسيولوجية مباشرة، تقنية ثنائية الدماغ.

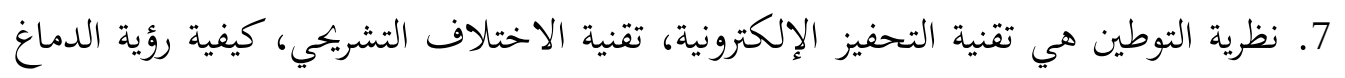
باستخدام PET (التصوير المقطعي بانبعاث البوز يترون).

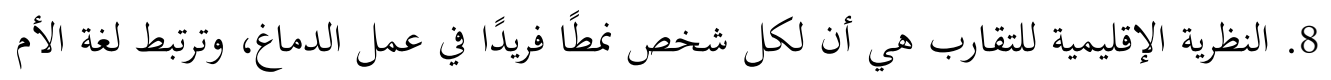

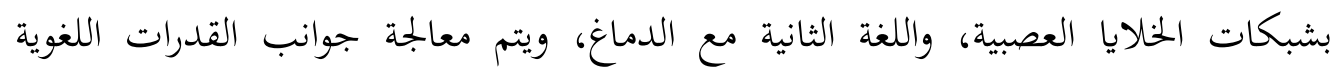

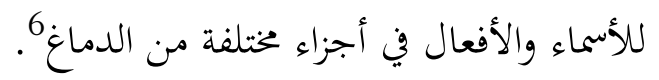

هـ. الو ظيفة العصبية للغة

إن نصفي الدماغ لمما أدوار مختلفة لوظيفة الككلام اللغوي المتمركزة في النصف الأيسر

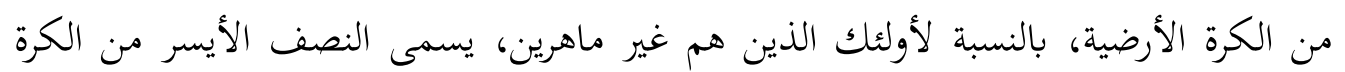

${ }^{5}$ Ahlsen, Elisabeth. 2006. Introduction to Neurolinguistics. Amsterdam: John Benjamins Publishing Company. Chomsky, Noam. 2000.

${ }^{6}$ Language, Thought, And the Brain. New York: Kluwer Academic Publishers 
الدماغية المهيمنة أو الفوقية الصوتية، وهو يختلف عن نصفي الكرة الأرضية غير المسيطر أو السفلي.

النصف الأيسر من الكرة الأرضية له معنى للغة بشكل رئيسي، كما أنه يعمل للذاكرة

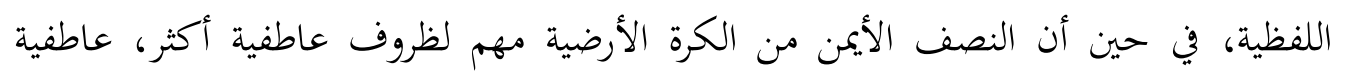

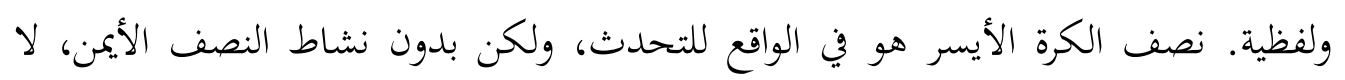

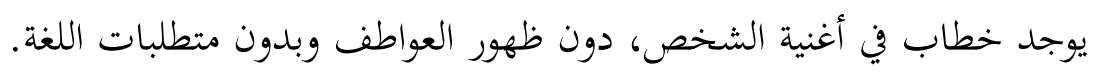

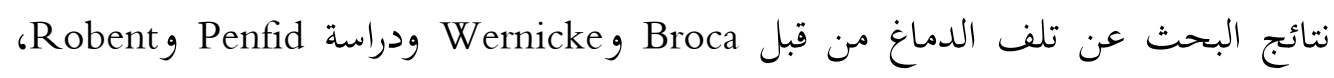
تؤدي إلى استنتاج مفاده أن نصف الكرة الأرضية متورط في العلاقات في وظيفة اللغة8 يقترح كريشن (1977) خمسة أسباب تكمن وراء هذا الاستنتاج، وهي:

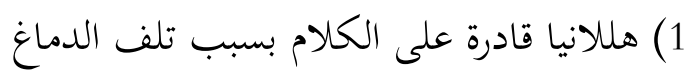

2) عندما يتم تخدير نصف الكرة الأيسر، تفقد القدرة على الكلام، ولكن يتم تخدير نصف ادلف الكرة الأيمن.

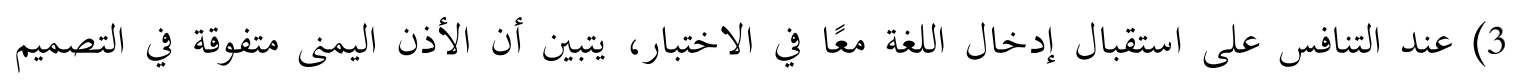

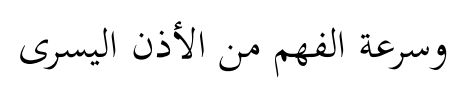

4) عندما يتم إعطاء مادة اللغة من خلال رؤية العين اليمنى والعين اليسرى، يتبين أن رؤية العين اليمنى اليسرى تكون أسرع وأكثر دقة في التقاط تلك المادة اللغوية من رؤية اليسار

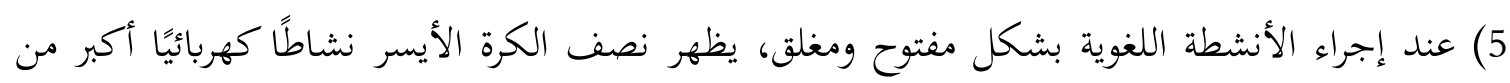

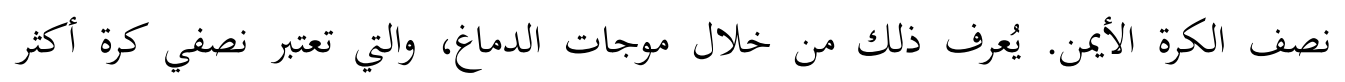

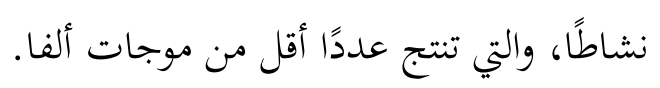

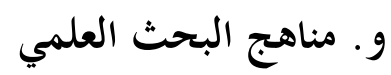

تمت كتابة هذه المقالة باستخدام طريقة البحث في المكتبة. البيانات المستخدمة كمصدر للكتابة

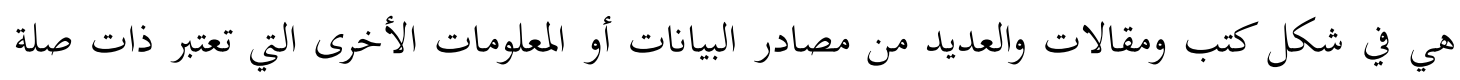
بالدراسة. وفي الوقت نفسه، في تحليل البيانات، يستخدم الكاتب طريقة التحليل الوصفي.

ز. الحملاصة

حدد هذا الفصل التمهيدي نطاق علم اللغة العصبي بتعريفه كدراسة للعلاقة بين الدماغ واللغة.

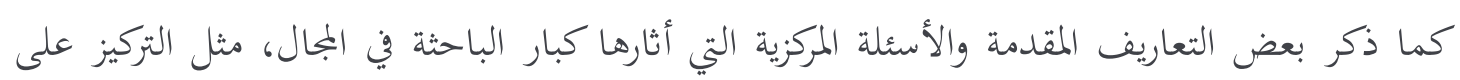
معالجة اللغة، وإشراك النماذج اللغوية النفسية التنبئية والتوضيحية، والدراسات التجريبية، والمحاكاة

7 البورين، عبد الرمحن أمحد، ها اللغة العربيَّة أصل اللغاتكل ، دار احلسن للنشر والتوزيع، ط1 ،1998. ${ }^{8}$ https://www.syr-res.com/article/18241.html

15 | الطموحات - السنة الثالثة، العدد الثاني، أغسطس 2020 available at: http://journal.uir.ac.id/index.php/THUMUHAT 
الحاسوبية، وقياس نشاط الدماغ أثناء المهام اللغوية. وعلاوة على ذلك فقد تم التأكيد على المكانة

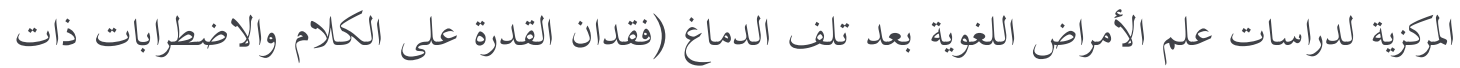

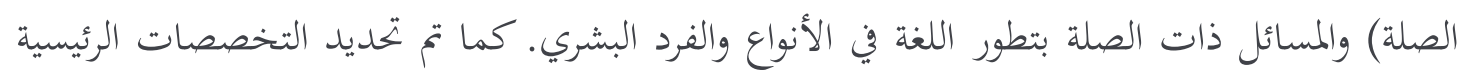
المساهمة التي تشمل علم اللغة، العلوم العصبية الطبية، علم النفس، وعلم أمراض النطق واللغة. الفصل أيضا بإيماز يوضح محتويات الكتاب. تلمراجع تدريب الرارى ص 5-6، نزهد النظر ص المداع

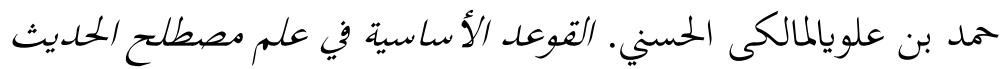

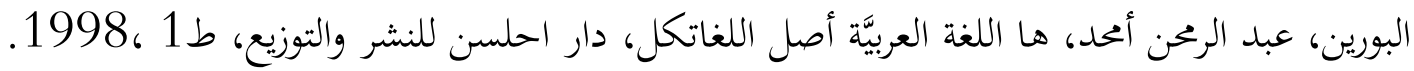

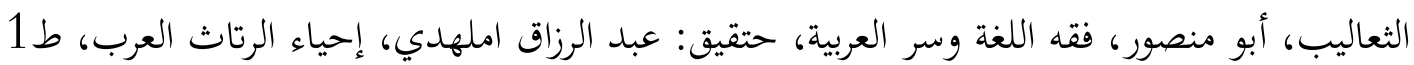
https://www.syr-res.com/article/18241.html

Elisabeth. Ahlsen 2006. Introduction To Neurolinguistics. Amsterdam: John Benjamins Publishing Company. Chomsky, Noam. 2000.

Horizon, News in The Study Of Language And Mind. America: Cambridge University Press. Glezerman, Tatyana, Victoria Balkoski. 2002.

Thought, Language, And the Brain. New York: Kluwer Academic Publishers 